\title{
A survey of nocturnal hypoxaemia and health related quality of life in patients with cryptogenic fibrosing alveolitis
}

\author{
M Clark, B Cooper, S Singh, M Cooper, A Carr, R Hubbard
}

\begin{abstract}
Background-A survey of overnight oximetry was conducted to estimate the prevalence of nocturnal hypoxaemia in patients with cryptogenic fibrosing alveolitis and to establish whether nocturnal hypoxaemia is related to quality of life. Methods-All patients with cryptogenic fibrosing alveolitis attending Nottingham City Hospital were invited to enter the study. Spirometric measurements and capillary blood gas tensions were obtained and overnight oxygen saturation was recorded at home. Quality of life was assessed using the Short Form-36, Chronic Respiratory Questionnaire, Hospital Anxiety Depression Scale, and Epworth Sleepiness Score questionnaires.

Results-Sixty seven eligible patients were identified and 50 agreed to enter the study, although two were subsequently excluded because they already used oxygen overnight. In the remaining 48 the mean (SD) overnight oxygen saturation $\left(\mathrm{SaO}_{2}\right)$ was $92.5(4.3) \%$ and the median number of dips greater than $4 \%$ per hour was 2.3 (interquartile range $1.5-5.3)$. Daytime oxygen level predicted mean overnight $\mathrm{SaO}_{2}(1.94 \% / \mathrm{kPa}, 95 \%$ CI 1.22 to 2.66 , $\mathbf{p}<0.001)$ but percentage predicted forced vital capacity (FVC) did not $(0.018 \% / \%$ predicted FVC, 95\% CI -0.04 to 0.08 , $\mathbf{p}=0.5$ ). Nocturnal hypoxaemia was associated with decreased energy levels and impaired daytime social and physical functioning, and these effects were independent of FVC.

Conclusions-Nocturnal hypoxaemia is common in patients with cryptogenic fibrosing alveolitis and may have an impact on health related quality of life. (Thorax 2001;56:482-486)
\end{abstract}

Keywords: cryptogenic fibrosing alveolitis; sleep; health related quality of life

Respiratory Medicine, Glenfield Hospital,

Leicester, UK

S Singh

Correspondence to: Dr R Hubbard

Richard.Hubbard@

Nottingham.ac.uk

Received 16 August 2000 Returned to authors 24 January 2001

Revised version received

14 February 2001

Accepted for publication

15 February 2001 been shown to improve symptoms of breathlessness ${ }^{67}$ and some patients have oxygen cylinders at home for this purpose. The use of long term oxygen therapy is currently only recommended on a short term basis for patients awaiting lung transplantation or when the resting daytime oxygen level is less than $8 \mathrm{kPa}^{7}$ Two small studies have shown that nocturnal hypoxaemia is common in the presence of severe lung fibrosis. ${ }^{89}$ Since nocturnal hypoxaemia may cause daytime symptoms, ${ }^{10}{ }^{11}$ nocturnal oxygen supplementation is a potential palliative treatment for patients with cryptogenic fibrosing alveolitis. As a prelude to an intervention study we have therefore estimated the prevalence of nocturnal hypoxaemia in patients with cryptogenic fibrosing alveolitis attending one hospital in Nottingham, and investigated whether nocturnal hypoxaemia has an impact on quality of life.

\section{Methods}

All patients with a diagnosis of cryptogenic fibrosing alveolitis attending Nottingham City Hospital were eligible to enter the study. Since only a few patients in the UK have an open lung biopsy ${ }^{12}$ we used our previous clinical criteria to define the diagnosis ${ }^{13}$ but, in addition, in this study patients with connective tissue disease were also included. Patients were identified using a combination of lung function records, hospital discharge codes, and our local cryptogenic fibrosing alveolitis database.

Following consent from the relevant general practitioner, we wrote to patients inviting them to take part in the study. Patients who agreed attended Nottingham City Hospital for one visit and, following a full explanation of the study, gave written consent. They attended the lung function laboratory for spirometric tests (dry bellows spirometer, Vitalograph Ltd, Bucks, UK) and, after 20 minutes at rest, capillary blood gas analysis and to complete health status questionnaires. The questionnaires used were the Short Form 36 (SF-36) ${ }^{14}$ to provide generic data on health status, the Epworth Sleepiness Score (EPSS) ${ }^{15}$ to provide a measure of daytime sleepiness, a self-completion version of the Chronic Respiratory Questionnaire (CRQ-SR) ${ }^{16}{ }^{17}$ to provide symptoms of respiratory disease, and the Hospital Anxiety Depression Scale (HADS) ${ }^{18}$ to provide a measure of anxiety and depression. In order to confirm the relevance of these questionnaires to patients with cryptogenic fibrosing alveolitis we carried out a brief qualitative study using a semi-structured interview to collect data from 
two patients with moderately severe disease. All questionnaires were administered by one of us (MC) who was blind to the lung function results. Nocturnal oxygen saturation $\left(\mathrm{SaO}_{2}\right)$ was measured using a Minolta Pulsox $3 i$ wrist oximeter which was worn by patients overnight at home and returned to us the next day. Data from the oximeters were downloaded onto an IBM compatible personal computer and analysed using software supplied by Minolta. If the probe attached time was less than 5 hours the test was deemed to be unsatisfactory and was repeated.

An initial descriptive analysis was performed to compare continuous variables using the unpaired $t$ test with prior log transformation of non-normally distributed variables. The main outcome variables were mean overnight $\mathrm{SaO}_{2}$ and the proportion of study time spent with an $\mathrm{SaO}_{2}$ of less than $90 \%$. Linear regression was used to establish the association between mean overnight $\mathrm{SaO}_{2}$ and both lung function measurements and health related quality of life measures, adjusting for the effects of age and sex. Nearly all subjects spent at least some of the night with an $\mathrm{SaO}_{2}$ of less than $90 \%$. When these data were examined graphically it was found that, for about $50 \%$ of the subjects, this proportion was short at less than $2 \%$. We therefore arbitrarily defined the sleep study as abnormal if more than $2 \%$ of the recording was spent at an $\mathrm{SaO}_{2}$ of less than $90 \%$ and used logistic regression to establish the association between the presence of an abnormal sleep study defined in this way and health related quality of life. Where consistent associations were found between health related quality of life outcomes and both measures of nocturnal hypoxaemia, these analyses were adjusted further for percentage predicted forced vital capacity (FVC) and were also repeated following exclusion of patients with a daytime oxygen level of less than $8 \mathrm{kPa}$.

The study was reviewed and approved by the Nottingham City Hospital ethics committee.

\section{Results}

Sixty seven eligible patients were identified of whom 50 agreed to take part in the study. Of the remaining 17,15 declined to take part (in five cases because they did not feel well enough) and we were unable to contact two. Of the 50 who did take part, $33(66 \%)$ were men, the mean (SD) age was 67.7 (8.7) years, and seven $(14 \%)$ had coexisting connective tissue disease. In each case the connective tissue disease was rheumatoid arthritis and these patients tended to be younger than patients with "lone" cryptogenic fibrosing alveolitis (61.6 $v 68.9$ years, $\mathrm{p}=0.04)$. The median time between date of diagnosis and recruitment was 2.1 years (interquartile range $1.0-4.4$ years). A high resolution $\mathrm{CT}$ scan was used to confirm the diagnosis in 40 patients $(80 \%)$. Subjects who did not have a CT scan tended to be those who were diagnosed longer ago (median time from diagnosis $8.0 v 1.7$ years, $\mathrm{p}=0.003)$ and those who were older (mean age 74.7 v 66.3 years, $\mathrm{p}=0.009)$. The mean $(\mathrm{SD})$ percentage

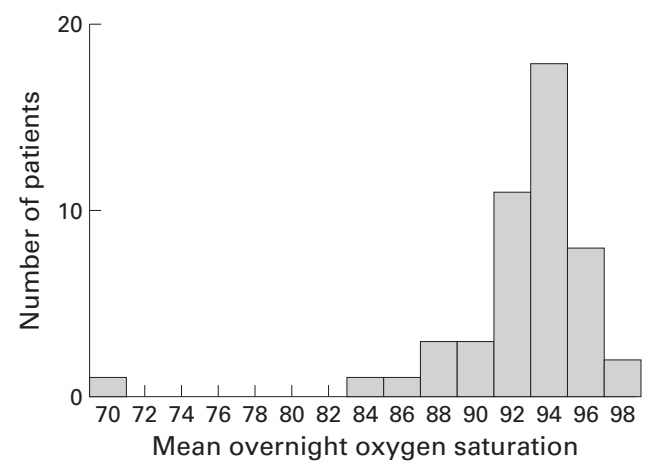

Figure 1 Distribution of mean overnight oxygen saturation values.

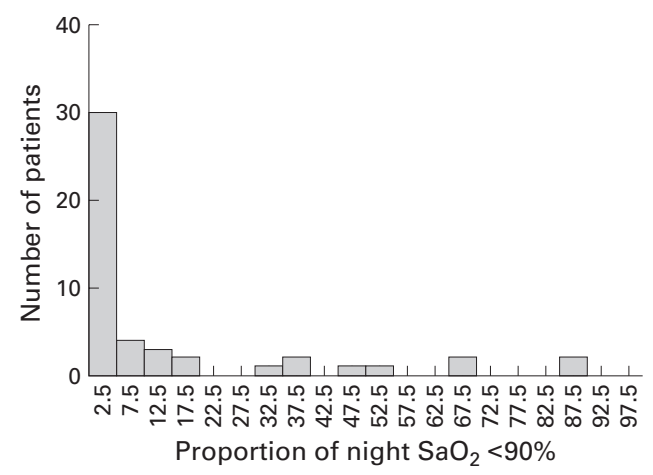

Figure 2 Distribution of proportion of time spent with an $\mathrm{SaO}_{2}$ of $<90 \%$.

predicted forced vital capacity (FVC) was 80.9 (25.0)\% and mean daytime capillary oxygen concentration was $9.3(1.6) \mathrm{kPa}$.

DAYTIME PHYSIOLOGICAL PREDICTORS OF NOCTURNAL HYPOXAEMIA

Two patients were already using oxygen at night and so were excluded from overnight oximetry measurements. In the remaining 48 none had a medical history suggestive of obstructive sleep apnoea. The mean duration of sleep recording was 7 hours and 53 minutes (range 5 hours 52 minutes to 10 hours 2 minutes) and the mean (SD) overnight $\mathrm{SaO}_{2}$ was 92.5 (4.3)\% (fig 1). Every subject had at least two dips in $\mathrm{SaO}_{2}$ of greater than $4 \%$, and the median number of dips per hour was 2.3 (interquartile range 1.5-5.3). A plot of the proportion of time spent with an $\mathrm{SaO}_{2}$ of less than $90 \%$ (fig 2) showed that just under half of the patients $(n=21)$ spent more than $2 \%$ of the night with an $\mathrm{SaO}_{2}$ of less than $90 \%$. Only four patients spent no time overnight with an $\mathrm{SaO}_{2}$ of less than $90 \%$. Age, sex, and percentage predicted FVC were not predictors of mean overnight $\mathrm{SaO}_{2}$ or the proportion of time spent with an $\mathrm{SaO}_{2}$ of less than $90 \%$, but resting daytime capillary oxygen level strongly predicted both (table 1).

RELATION BETWEEN PERCENTAGE PREDICTED FVC AND HEALTH RELATED QUALITY OF LIFE

Within the CRQ-SR questionnaire domains we found evidence of a strong association between percentage predicted FVC and mastery and a weaker association with dyspnoea (table 2). For the SF-36 the strongest association was found 
Table 1 Age, sex, focred vital capacity (FVC), and daytime oxygen level ( $\left.\mathrm{SaO}_{2}\right)$ as predictors of nocturnal hypoxaemia

\begin{tabular}{|c|c|c|c|c|c|c|}
\hline & \multicolumn{3}{|c|}{ Mean overnight $\mathrm{SaO}_{2}$} & \multicolumn{3}{|c|}{$\begin{array}{l}\text { Abnormal sleep study (more than } \\
2 \% \text { of time with } \mathrm{SaO}_{2}<90 \% \text { ) }\end{array}$} \\
\hline & $\begin{array}{l}\text { Regression } \\
\text { coefficient }\end{array}$ & $95 \% C I$ & $p$ value & $\begin{array}{l}\text { Odds } \\
\text { ratio }^{*}\end{array}$ & $95 \% C I$ & $p$ value \\
\hline Sex & -0.37 & -3.04 to 2.31 & 0.8 & 1.24 & 0.38 to 4.11 & 0.7 \\
\hline Age & -0.04 & -0.19 to 0.10 & 0.6 & 1.01 & 0.95 to 1.08 & 0.7 \\
\hline \multicolumn{7}{|l|}{ FVC } \\
\hline (\% predicted) & 0.018 & -0.04 to 0.08 & 0.5 & 0.99 & 0.97 to 1.02 & 0.6 \\
\hline $\begin{array}{l}\text { Capillary oxygen } \\
\text { concentration }\end{array}$ & 1.94 & 1.22 to 2.66 & $<0.001$ & 0.46 & 0.26 to 0.82 & 0.002 \\
\hline
\end{tabular}

${ }^{\star}$ Regression coefficients are for the change in mean overnight $\mathrm{SaO}_{2}$ predicted by women $v$ men and for each increase in year of age, percentage predicted $\mathrm{FVC}$, and $\mathrm{kPa}$ of daytime oxygen. Regression coefficients and odds ratios were all adjusted for the effects of age and sex.

Table 2 Relation of health related quality of life to percentage predicted forced vital capacity (FVC)

\begin{tabular}{llll}
\hline & $\begin{array}{l}\text { Regression } \\
\text { coefficient }\end{array}$ & $95 \%$ CI & p value \\
\hline CRQ-SR & 5.28 & -0.19 to 10.75 & 0.06 \\
Dyspnoea & 4.11 & -0.83 to 9.04 & 0.1 \\
Fatigue & 3.14 & -2.06 to 8.34 & 0.2 \\
Emotion & 7.15 & 2.87 to 11.42 & 0.002 \\
Mastery & & & \\
SF-36 & 1.32 & 0.13 to 2.51 & 0.03 \\
Physical functioning & 1.91 & -3.77 to 7.59 & 0.5 \\
Role physical & 3.58 & -1.98 to 9.14 & 0.2 \\
Role mental & 1.87 & -0.74 to 4.48 & 0.2 \\
Social functioning & 1.51 & -1.42 to 4.44 & 0.3 \\
Pain & 1.17 & -0.11 to 2.44 & 0.07 \\
Mental health & 1.91 & 0.35 to 3.47 & 0.02 \\
Energy & 2.67 & 1.16 to 4.18 & 0.001 \\
Health perception & & & \\
& & -2.97 to 0.34 & 0.1 \\
HADS & -1.31 & -3.51 to -0.06 & 0.04 \\
Anxiety & & & \\
Depression & -1.79 & -2.14 to 1.03 & 0.5 \\
EPSS & -0.56 &
\end{tabular}

CRQ-SR = Chronic Respiratory Questionnaire; SF-36 = Short Form 36; HADS = Hospital Anxiety Depression Scale; EPSS = Epworth Sleepiness Score.

${ }^{\star}$ Regression coefficients are for change in percentage predicted FVC predicted by a unit increase in questionnaire score. The regression coefficients are adjusted for age and sex.

between health perception and percentage predicted FVC, although there was also some evidence of an association with the physical functioning, mental health, and energy domains. For the HADS questionnaire there was some evidence of an association with depression but not anxiety. There was no association between the Epworth sleepiness score and percentage predicted FVC.

RELATION BETWEEN NOCTURNAL HYPOXAEMIA AND HEALTH RELATED QUALITY OF LIFE Within the domains of the CRQ-SR there was some evidence of an association between mastery and mean overnight $\mathrm{SaO}_{2}$ but not with the presence of an abnormal sleep study as defined by the proportion of time spent with an $\mathrm{SaO}_{2}$ of less than $90 \%$ (table 3). Within the SF-36 scores the physical functioning, social functioning, and energy domains were associated with lower overnight $\mathrm{SaO}_{2}$ and an abnormal sleep study. The strongest associations were with the energy domain, and these were essentially unchanged by adjusting for percentage predicted FVC (adjusted regression coefficient $0.41 \%$ per questionnaire score, $95 \%$ CI 0.12 to 0.71 , adjusted odds ratio $0.77,95 \%$ CI 0.64 to 0.93) and, although attenuated, remained when the analysis was limited to patients with a daytime $\mathrm{SaO}_{2}$ of more than $8 \mathrm{kPa}$ (regression coefficient $0.26 \%$ per questionnaire score, $95 \%$ CI 0.03 to 0.50 , odds ratio $0.80,95 \%$ CI 0.64 to 1.01). The effect of adjusting for percentage predicted FVC and restricting the analysis to patients with a daytime oxygen of more than $8 \mathrm{kPa}$ was similar for the physical functioning and social functioning domains. There was no consistent evidence for an association between nocturnal hypoxaemia and the anxiety or depression domains of the HADS questionnaire. The presence of an abnormal sleep study was related to the Epworth sleepiness score but there was no association with mean overnight $\mathrm{SaO}_{2}$.

\section{Discussion}

The results of this study show that nocturnal hypoxaemia is common in patients with cryptogenic fibrosing alveolitis attending hospitals in the UK. The best physiological

Table 3 Relation between health related quality of life and nocturnal hypoxaemia

\begin{tabular}{|c|c|c|c|c|c|c|}
\hline & \multicolumn{3}{|c|}{ Mean overnight $\mathrm{SaO}_{2}$} & \multicolumn{3}{|c|}{ Abnormal sleep study } \\
\hline & $\begin{array}{l}\text { Regression } \\
\text { coefficient }^{*}\end{array}$ & $95 \% C I$ & $p$ value & Odds ratio * & $95 \% C I$ & $p$ value \\
\hline \multicolumn{7}{|l|}{ CRQ-SR } \\
\hline Dyspnoea & 0.38 & -0.64 to 1.40 & 0.5 & 0.75 & 0.46 to 1.23 & 0.3 \\
\hline Fatigue & 0.80 & -0.17 to 1.75 & 0.1 & 0.67 & 0.42 to 1.08 & 0.1 \\
\hline Emotion & 0.50 & -0.61 to 1.61 & 0.4 & 0.91 & 0.57 to 1.47 & 0.7 \\
\hline Mastery & 1.15 & 0.28 to 2.02 & 0.01 & 0.80 & 0.52 to 1.22 & 0.3 \\
\hline \multicolumn{7}{|l|}{ SF-36 } \\
\hline Physical functioning & 0.23 & 0.00 to 0.46 & 0.05 & 0.87 & 0.77 to 0.99 & 0.03 \\
\hline Role physical & 0.60 & -0.41 to 1.60 & 0.2 & 0.71 & 0.43 to 1.17 & 0.2 \\
\hline Role mental & -0.16 & -1.19 to 0.86 & 0.8 & 0.77 & 0.49 to 1.22 & 0.3 \\
\hline Social functioning & 0.60 & 0.14 to 1.05 & 0.01 & 0.77 & 0.60 to 0.98 & 0.03 \\
\hline Pain & 0.11 & -0.42 to 0.63 & 0.7 & 0.88 & 0.69 to 1.11 & 0.3 \\
\hline Mental health & 0.22 & -0.01 to 0.44 & 0.06 & 0.94 & 0.85 to 1.05 & 0.3 \\
\hline Energy & 0.41 & 0.14 to 0.68 & 0.004 & 0.77 & 0.65 to 0.92 & 0.005 \\
\hline Health perception & 0.15 & -0.16 to 0.46 & 0.3 & 0.84 & 0.72 to 0.99 & 0.04 \\
\hline \multicolumn{7}{|l|}{ HADS } \\
\hline Anxiety & -0.05 & -0.25 to 0.15 & 0.6 & 1.04 & 0.91 to 1.19 & 0.6 \\
\hline Depression & -0.19 & -0.49 to 0.12 & 0.3 & 1.13 & 0.97 to 1.32 & 0.1 \\
\hline EPSS & -0.15 & -0.43 to 0.14 & 0.3 & 1.27 & 1.07 to 1.52 & 0.006 \\
\hline
\end{tabular}

CRQ-SR = Chronic Respiratory Questionnaire; SF-36 = Short Form 36; HADS = Hospital Anxiety Depression Scale; EPSS = Epworth Sleepiness Score; $\mathrm{SaO}_{2}=$ oxygen saturation.

${ }^{\star}$ Regression coefficients are presented for each change in mean overnight $\mathrm{SaO}_{2}$ predicted by each unit increase in questionnaire score. All regression coefficients and odds ratios are adjusted for age and sex. 
predictor of nocturnal hypoxaemia was resting daytime oxygen level while, in contrast, FVC was a poor predictor. On the basis of the questionnaires used, nocturnal hypoxaemia appears to be associated with a reduction in energy levels and impairment of physical and social functioning, and these effects are independent of FVC.

In this study the aim was to recruit patients who were representative of those with cryptogenic fibrosing alveolitis being cared for by chest and general physicians in the UK. For this reason we tried to include all patients, including those with coexisting connective tissue disease, and chose to study nocturnal hypoxaemia at home using simple wrist pulse oximeters which are easy to use, cheap, and convenient for patients. Patients with coexisting connective tissue disease may have changes in health related quality of life outcomes because of symptoms resulting from the comorbid illness. However, comorbidity is also common in patients with "lone" cryptogenic fibrosing alveolitis-particularly heart disease $^{1920}$ and lung cancer ${ }^{192122}$ - so we decided not to exclude patients with additional illnesses. In fact, the results of the study were similar when the analysis was restricted to patients with "lone" cryptogenic fibrosing alveolitis. The use of additional sleep studies on consecutive nights would have been beneficial in giving an assessment of night-to-night variation in nocturnal hypoxia but, in order to keep the study simple for patients and more relevant to clinical practice, we did not do this.

Since our study is cross sectional it includes only prevalent cases so patients with a better prognosis will tend to be over-represented ${ }^{1}$ as those who die rapidly are not under long term follow up. Therefore, although we tried to include patients with the full spectrum of disease severity, our sample is likely to be made up of patients with a better prognosis than a cohort of newly diagnosed cases. This bias is unlikely to alter the physiological finding that nocturnal hypoxaemia is common in patients with cryptogenic fibrosing alveolitis and that daytime oxygen concentration is the best predictor of nocturnal hypoxaemia, but it may lead to some dilution of the associations between physiological measures and health related quality of life scores because, with time, patients will reset their goals and develop coping mechanisms.

The strong association between daytime oxygen levels and nocturnal hypoxaemia is not surprising. In a study of 11 patients with severe interstitial lung disease ${ }^{9}$ the five patients with a daytime $\mathrm{SaO}_{2}$ of less than $90 \%$ had a significantly lower overnight $\mathrm{SaO}_{2}$ than the six with a daytime $\mathrm{SaO}_{2}$ of more than $90 \%$. Furthermore, previous studies in patients with chronic obstructive pulmonary disease (COPD) have shown that mean awake $\mathrm{SaO}_{2}$ is a strong predictor of both mean and lowest overnight $\mathrm{SaO}_{2} .{ }^{10}{ }^{23}$ In patients with COPD who have nocturnal hypoxaemia the main mechanism appears to be hypoventilation, particularly during periods of rapid eye movement sleep
$(\mathrm{REM})^{10}$ and, as for our patients with cryptogenic fibrosing alveolitis, daytime spirometric parameters are poor predictors of nocturnal $\mathrm{SaO}_{2}$.

The measures of health related quality of life suggest that nocturnal hypoxaemia leads to decreased daytime energy levels and impaired social and physical functioning, and that these effects are independent of FVC. Although FVC was associated with the dyspnoea domain of the CRQ-SR, neither were associated with nocturnal hypoxaemia. The most obvious explanation for these findings is that nocturnal hypoxaemia disturbs the sleep patterns of patients with cryptogenic fibrosing alveolitis and that this has an impact on daytime health related quality of life. One previous study has shown that patients with severe interstitial lung disease have fragmented sleep and spend more time in stage 1 sleep and less time in REM sleep. ${ }^{9}$ The alternative explanation is that the associations we found are due to disease severity in general, and that nocturnal hypoxaemia is merely another marker of disease severity not adequately captured by FVC. Ultimately, this issue can only be resolved by studying the efficacy of nocturnal oxygen supplementation on health related quality of life in patients with cryptogenic fibrosing alveolitis. Our finding of associations between nocturnal hypoxaemia and health related quality of life for patients with a daytime oxygen level of more than $8 \mathrm{kPa}$ - that is, patients for whom long term oxygen therapy is not currently recommended ${ }^{7}$ - suggests that such a trial should include the full spectrum of patients with cryptogenic fibrosing alveolitis.

In summary, nocturnal hypoxaemia is a common problem in patients with cryptogenic fibrosing alveolitis and is associated with decreased daytime energy levels and impaired social and physical functioning independent of the level of FVC. Daytime oxygen level is the best predictor of nocturnal hypoxaemia and FVC is a poor predictor. Overnight oxygen supplementation is a potential palliative treatment for patients with cryptogenic fibrosing alveolitis and should be assessed in a randomised clinical trial

The authors would like to thank lung function technicians Sue Hazard, Philomena Dennis, Julie Christian, Rachel Anthony and Karen Lakin, the City Hospital Chaplaincy and, in particular, Martin Kerry for allowing them to use a quiet room for the study.

Funding: Trent National Health Service Research and Development Project Grant.

1 Hubbard R, Johnston I, Britton J. Survival in patients with ryptogenic fibrosing alveolitis: a population based cohort study. Chest 1998;113:396-400.

2 British Thoracic Society. The diagnosis, assessment and treatment of diffuse parenchymal lung disease in adults. Thorax 1999;54(Suppl 1):S1-30.

3 Raghu G, Depaso WJ, Cain K, et al. Azathioprine combined with prednisone in the treatment of idiopathic pulmonary fibrosis: a prospective double-blind, randomized, placebofibrosis: a prospective double-blind, randomized, placebo4 controlled clinical trial. Am Rev Respir Dis 1991;144:291-6. Johnson MA, Kwan S, Snell NJ, et al. Randomised controlled trial comparing prednisolone alone with cyclophosphamide and low dose prednisolone in combination

5 Turner-Warwick M, Burrows B, Johnson A. Cryptogenic fibrosing alveolitis: response to corticosteroid treatment fibrosing alveolitis: response to corticosteroid 
6 Swinburn CR, Mould H, Stone TN, et al. Symptomatic benefit of supplemental oxygen in hypoxemic patients with .

7 Working Party of the Royal College of Physicians. Domiciliary oxygen therapy services: clinical guidelines and advice for prescribers. London: Royal College of Physicians of London, 1999.

8 Bye PTB, Issa F, Berthon-Jones $\mathrm{M}$, et al. Studies of oxygenation during sleep in patients with interstitial lung disease. Am Rev Respir Dis 1984;129:27-32.

9 Perez-Padilla R, West P, Lertzman M, et al. Breathing during sleep in patients with interstitial lung disease. Am Rev Respir Dis 1985;132:224-9.

10 Douglas NJ, Flenley DC. Breathing during sleep in patients with obstructive lung disease. Am Rev Respir Dis 1990;141: 1055-70.

11 Stradling JR. Sleep apnoea syndromes. In: Brewis RAL, Corrin B, Geddes DM, Gibson GJ, eds. Respiratory

medicine. 2 nd ed. Lon Johnston IDA, Gomm SA, Kalra A, et al. The management of cryptogenic fibrosing alveolitis in three regions of the
United Kingdom. Eur Respir f 1993;6:891-3.

United Kingdom. Eur Respir f 1993;6:891-3.

13 Hubbard R, Lewis S, Richards K, et al. Occupational exposure to metal or wood dust and aetiology of cryptogenic fibrosing alveolitis. Lancet 1996;347:284-9.

14 Jenkinson C, Layte R, Wright L, et al. The UK SF-36: an analysis and interpretation manual. Oxford: University of Oxford, 1996.
15 Johns MW. Daytime sleepiness, snoring and obstructive sleep apnea: the Epworth sleepiness scale. Chest 1993;103: 30-6.

16 Guyatt GH, Berman L, Townsend M. A measure of quality of life for clinical trials in chronic lung disease. Thorax 1987;42:773-8.

17 WIlliams J, Singh S, Morgan MDL. A comparison between a self-reported Chronic Respiratory Disease Questionnaire (CRQ-SR) and the conventional interviewer led CRQ Eur Respir F 1999;14:118s.

18 Zigmond AS, Snaith RP. The Hospital Anxiety Depression Scale. Acta Psychiatr Scand 1983;67:361-70.

19 Panos RJ, Mortenson RL, Niccoli SA, et al. Clinical deterioration in patients with idiopathic pulmonary fibrosis: causes and assessment. Am F Med 1990;88:396-404.

20 Turner-Warwick M, Burrows B, Johnson A. Cryptogenic fibrosing alveolitis: clinical features and their influence on survival. Thorax 1980;35:171-80.

21 Hubbard R, Venn A, Lewis S, et al. Lung cancer and cryptogenic fibrosing alveolitis: a population based cohort study. Am f Respir Crit Care Med 2000;161:5-8.

22 Turner-Warwick M, Lebowitz $\mathrm{M}$, Burrows B, et al. Cryptogenic fibrosing alveolitis and lung cancer. Thorax 1980;35:496-9.

23 Cormick W, Olson LG, Hensley MJ, et al. Nocturnal hypoxaemia and quality of sleep in patients with chronic obstructive pulmonary disease. Thorax 1986;41:846-54. 